\title{
Preparing the exoplanet search with PRIMA: searching for reference stars and target characterization
}

\author{
R. Geisler ${ }^{1,2, *}$, J. Setiawan ${ }^{2}$, Th. Henning ${ }^{2}$, \\ D. Queloz ${ }^{3}$, A. Quirrenbach ${ }^{1}$, R. Launhardt ${ }^{2}$, \\ A. Müller ${ }^{2}$, S. Reffert ${ }^{1}$, P. Weise ${ }^{2}$ and ESPRI consortium ${ }^{1,2,3,4}$ \\ ${ }^{1}$ ZAH - Landessternwarte, Königstuhl 12, D-69117 Heidelberg, Germany \\ ${ }^{2}$ Max-Planck-Institut für Astronomie, Königstuhl 17, D-69117 Heidelberg, Germany \\ ${ }^{3}$ Observatoire Astronomique de l'Université de Genève, CH-1290 Sauverny, Switzerland \\ ${ }^{4}$ European Southern Observatory (ESO), D-85748 Garching, Germany \\ * Fellow of the IMPRS for Astronomy and Cosmic Physics at the \\ University of Heidelberg, email: rgeisler@lsw.uni-heidelberg.de
}

\begin{abstract}
The PRIMA (Phase-Referenced Imaging and Micro-arcsecond Astrometry) facility at ESO VLTI (Paranal observatory) is expected to be commissioned in mid 2008. The ESPRI (Exoplanet Search with PRIMA) consortium is currently preparing an astrometric survey to search for extrasolar planets. To achieve the scientific goal of this survey, a careful selection of target and reference stars is necessary. Apart from catalog search and modelling, extensive and dedicated preparatory observations are indispensable. Here we present two aspects of the preparatory observation programs: A high dynamic range near infrared (NIR) imaging survey to search for astrometric reference stars around the preselected target stars and characterization of the target stars by using high-resolution spectroscopy.
\end{abstract}

Keywords. astrometry, stars: planetary systems, techniques: interferometric, photometric, spectroscopic, radial velocities

\section{Introduction}

Relative astrometry with an accuracy of $10 \mu \operatorname{arcsec}$ with PRIMA by using interferometric delays relies on phase reference stars at small angular separation (within 10 to 15 arcsec) from the target stars. Therefore, to prepare our planet search program ESPRI (Launhardt et al. 2008a/b), a dedicated search for reference stars around our potential planet-search targets is mandatory. In addition, accurate determination of fundamental stellar parameters and characterization of the stellar activity are useful to select appropriate target stars for ESPRI.

For this reason the usefulness of available catalogs in various archives was tested. The limiting magnitude in K-band with PRIMA and the Auxiliary Telescopes (ATs) for the bright target star will be $\mathrm{K} \approx 12 \mathrm{mag}$ and for the fainter reference star $\mathrm{K} \approx 14-16 \mathrm{mag}$. Due to saturations and artifacts of the bright star, it is not possible to identify faint potential reference stars close to the bright star with the required brightness contrast in the available catalogs (e.g. 2MASS: Figure 1). This makes the majority of available catalogs useless for this task and an additional high dynamic range NIR imaging survey is necessary. In these proceedings we present the preliminary results of our NIR imaging survey and target characterization by high-resolution spectroscopy. 

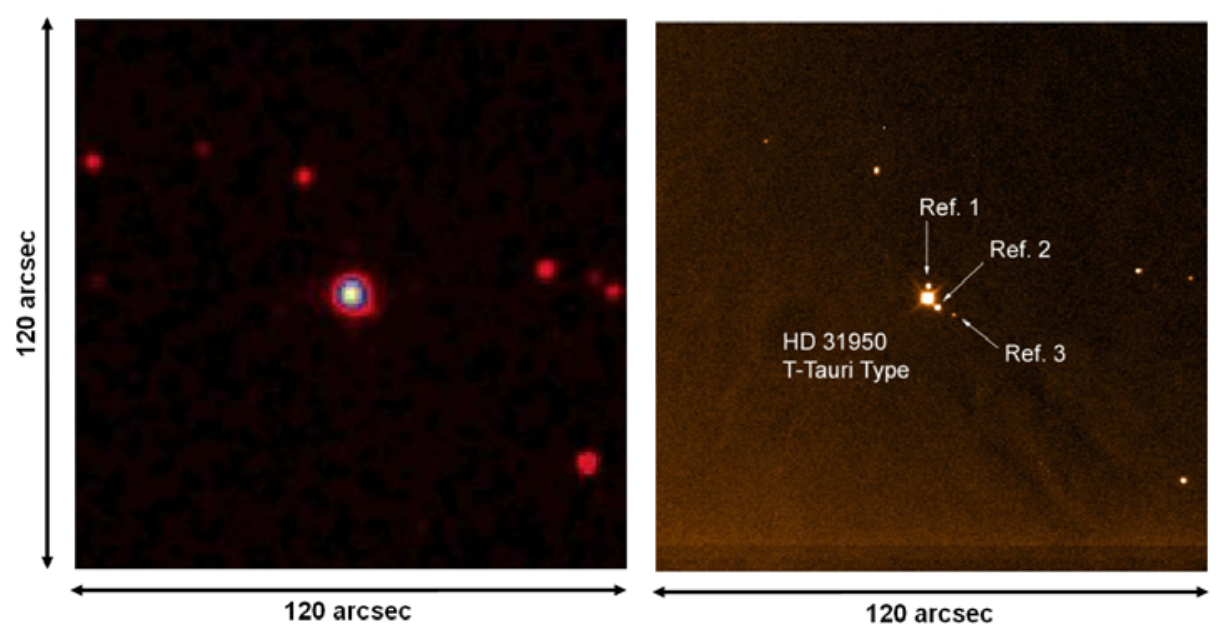

Figure 1. K-band image of HD 31950 obtained with SOFI (right panel). This star of T-Tauri type is part of the young star group. Three phase reference stars are found within 10 arcsec of the target star. In the contrast enhanced 2MASS K-band image no close reference stars are visible (left panel).

\section{Observations and Preliminary Results}

For our NIR imaging observations we used SOFI at the NTT (ESO, La Silla) and OMEGA Cass at the $3.5 \mathrm{~m}$ telescope (Calar Alto observatory). To achieve the required limiting magnitude for the astrometric reference stars we searched down to a detection limit of $\mathrm{K} \approx 17 \mathrm{mag}$. To characterize the reference stars (e.g., spectral type, photometric distance) all target stars with identified reference stars are additionally observed in Jband. The spectroscopic observations were done by using FEROS $(R=48000)$ at the 2.2 m MPG/ESO telescope in La Silla.

The ESPRI target list consists currently of nearby stars (main-sequence stars within $15 \mathrm{pc}$ ), young stars (within $100 \mathrm{pc}$ and ages 5...300 Myr) and stars with known radial velocity planets, and contains 865 stars altogether. Almost half of of them have been observed already with SOFI and OMEGA Cass. The analysis of the images has been completed. We found at least one reference star within 10 arcsec and with $\mathrm{K} \leqslant 16$ mag in $22 \%$ of all observed targets (Table 1 ). The rate predicted by modelling is $20 \%$ (Figure

\begin{tabular}{|c|c|c|c|c|c|c|}
\hline \multirow{2}{*}{$\begin{array}{l}\text { Star } \\
\text { group }\end{array}$} & \multirow{2}{*}{$\begin{array}{l}\text { Number } \\
\text { of stars }\end{array}$} & \multicolumn{2}{|c|}{ Observed } & \multicolumn{2}{|c|}{ Reference stars found (K $\leq 16 \mathrm{mag})$} & \multirow{2}{*}{$\frac{2 \text { MASS }}{2^{\prime \prime} \cdot 10^{\prime \prime}}$} \\
\hline & & SOFI & OMEGA Cass & $2^{\prime \prime}-10^{\prime \prime}$ & $2^{\prime \prime}-20^{\prime \prime}$ & \\
\hline Nearby Stars & 336 & \multirow{3}{*}{163} & \multirow{3}{*}{259} & \multirow{3}{*}{91} & \multirow{3}{*}{148} & $0.6 \%$ \\
\hline Young Stars & 381 & & & & & $11.8 \%$ \\
\hline Radial Velocity & 148 & & & & & $8.8 \%$ \\
\hline Total: & 865 & \multicolumn{2}{|c|}{$49 \%$} & $22 \%$ & $35 \%$ & $7.0 \%$ \\
\hline
\end{tabular}

Table 1. 422 of 865 stars (49\%) from our target list have been observed with SOFI and OMEGA Cass already. Out of these we found 91 stars $(22 \%)$ with at least one reference star between 2 and 10 arcsec with $\mathrm{K} \leqslant 16$ mag. Reference stars with separations between 10 and 20 arcsec are still useful for lower anticipated accuracy and thus would still allow the detection of a giant planet. 


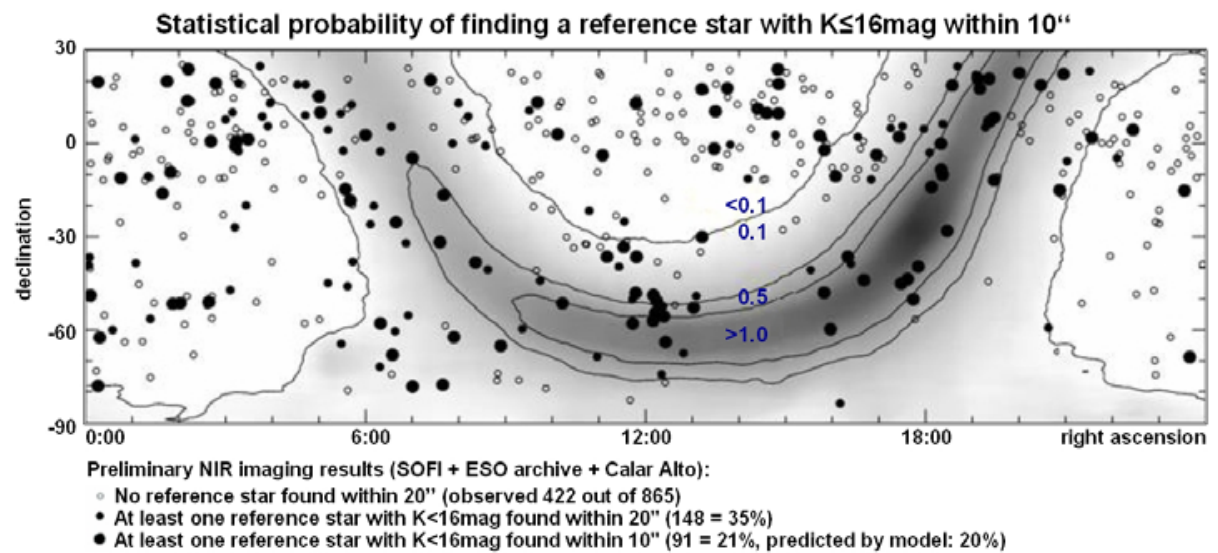

Figure 2. Sky map showing the statistical probability of finding at least one reference star with $\mathrm{K} \leqslant 16 \mathrm{mag}$ within 10 arcsec around any given star (grey scale: less then $10 \%$ to $100 \%$ ). The map was constructed from COBE DIRBE NIR all sky maps and calibrated with Besançon synthetic stellar population models. Plotted as dots are the results from our NIR observations. The actual detection rates agree with the predicted ones to within $2 \%$, thus proving the applicability and usefulness of the model.

2 ) and very close to the actual detection rate. Only $7 \%$ of the stars have a reference star with the required properties in 2MASS.

From the spectroscopic observations we determined the fundamental parameters of the target stars. In addition, we also monitor radial velocity variations of the targets to identify possible stellar/substellar companions or starspots. In case of stellar companions and starspots, the targets should be examined carefully whether they are still suitable for the anticipated astrometric observations. In case of substellar companions (planets/brown dwarfs), those targets with available reference stars are of high-priority to be observed with PRIMA. Both results, from NIR imaging and spectroscopy, will be included in a dedicated target database (ASTRID).

\section{ASTRID Database (http://www.mpia-hd.mpg.de/ASTRID/)}

ASTRID (AStrometric Target and Reference stars Interactive Database) was developed to collect relevant parameters for the target and reference star pairs. The database includes archival data (basically from Hipparcos and 2MASS) on the stars as well as the measurements obtained from the preparatory programs (NIR imaging, spectroscopy and astrometry). Parts of the database will be publicly accessible. When ESPRI observations commence with the VLTI, astrometric results will also be added to the ASTRID database.

\section{References}

Launhardt, R., A., Henning, Queloz, D., Quirrenbach, T., Elias, N., Pepe, F., Reffert, S., Segransan, D., Setiawan, J., \& ESPRI Consortium 2008a, The ESPRI project: Astrometric Exoplanet Search with PRIMA, These Proceedings.

Launhardt, R., A., Henning, Queloz, D., Quirrenbach, T., Elias, N., Pepe, F., Reffert, S., Segransan, D., Setiawan, J., \& ESPRI Consortium 2008b, The ESPRI project: Narrow-angle astrometry with VLTI-PRIMA, Proc. IAUS, 248, in press. 